\title{
Monogenic diabetes in overweight and obese youth diagnosed with type 2 diabetes: the TODAY clinical trial
}

\author{
Jeffrey W. Kleinberger, $\mathrm{PhD}^{1}$, Kenneth C. Copeland, $\mathrm{MD}^{2}$, Rachelle G. Gandica, $\mathrm{MD}^{3}$, \\ Morey W. Haymond, MD ${ }^{4}$, Lynne L. Levitsky, MD ${ }^{5}$, Barbara Linder, MD, PhD ${ }^{6}$, Alan R. Shuldiner, MD ${ }^{1,7}$, \\ Sherida Tollefsen, MD ${ }^{8}$, Neil H. White, MD ${ }^{9}$ and Toni I. Pollin, MS, PhD ${ }^{1}$; for the TODAY Study Group ${ }^{10}$
}

Purpose: Monogenic diabetes accounts for 1-2\% of diabetes cases. It is often undiagnosed, which may lead to inappropriate treatment. This study was performed to estimate the prevalence of monogenic diabetes in a cohort of overweight/obese adolescents diagnosed with type 2 diabetes (T2D).

Methods: Sequencing using a custom monogenic diabetes gene panel was performed on a racially/ethnically diverse cohort of 488 overweight/obese adolescents with T2D in the Treatment Options for Type 2 Diabetes in Adolescents and Youth (TODAY) clinical trial. Associations between having a monogenic diabetes variant and clinical characteristics and time to treatment failure were analyzed.

Results: More than $4 \%(22 / 488)$ had genetic variants causing monogenic diabetes (seven GCK, seven HNF4A, five HNF1A, two INS, and one KLF11). Patients with monogenic diabetes had a statistically, but not clinically, significant lower body mass index (BMI) $z$-score, lower fasting insulin, and higher fasting glucose. Most (6/7) patients with HNF4A variants rapidly failed TODAY treatment across study arms (hazard ratio $=5.03, P=0.0002$ ), while none with GCK variants failed treatment.

Conclusion: The finding of $4.5 \%$ of patients with monogenic diabetes in an overweight/obese cohort of children and adolescents with T2D suggests that monogenic diabetes diagnosis should be considered in children and adolescents without diabetes-associated autoantibodies and maintained C-peptide, regardless of BMI, as it may direct appropriate clinical management.

Genet Med advance online publication 12 October 2017

Key Words: clinical trial; monogenic diabetes; obesity; type 2 diabetes; youth

\section{INTRODUCTION}

Maturity-onset diabetes of the young (MODY), the most common category of monogenic diabetes, results from a single variant in an individual patient in one of 14 known genes. In the 1970s, the term MODY was created to characterize patients described by Stefan Fajans as having a non-insulin-dependent form of diabetes at a young age. ${ }^{1}$ (Since then, it has been suggested that "MODY" be changed to "familial young-onset diabetes" because of the discovery of multiple causative genes and a better understanding of the gene-specific patient characteristics combined with the increased prevalence of type 2 diabetes (T2D) in childhood. ${ }^{2}$ While this nomenclature change is appropriate, the term MODY is used in this article to differentiate from other forms of monogenic diabetes, such a neonatal or syndromic forms of monogenic diabetes, and because the term is well recognized by the general population.) Epidemiological studies across Europe determined that MODY accounts for approximately $1-2 \%$ of all diabetes. ${ }^{3}$ GCK-MODY, HNF1A-MODY, and
HNF4A-MODY account for at least $85 \%$ of MODY cases. ${ }^{4-6}$ MODY generally presents in an autosomal-dominant pattern of inheritance early in life as non-insulin-requiring hyperglycemia. Correct characterization of monogenic diabetes is important for optimal patient treatment since the most common etiologies can be effectively treated with methods different from first-line treatments for type 1 diabetes (T1D) (insulin) or T2D (metformin). Patients with HNF1A-MODY and HNF4A-MODY are effectively treated with oral sulfonylurea therapy. ${ }^{7,8}$ Patients with GCK-MODY have mildly elevated baseline blood glucose concentrations that commonly do not require treatment and do not lead to diabetic complications. ${ }^{9}$ Therefore, proper diagnosis of monogenic diabetes can lead to more effective, less invasive, and less expensive treatment for patients and potentially family members with the same variant.

American Diabetes Association guidelines suggest that a diagnosis of monogenic diabetes be considered when diabetes is diagnosed in the first 6 months of life, when the patient

\footnotetext{
${ }^{1}$ Division of Endocrinology, Diabetes, and Nutrition, Department of Medicine, University of Maryland School of Medicine, Baltimore, Maryland, USA; ${ }^{2}$ Department of Pediatrics, University of Oklahoma College of Medicine, Oklahoma City, Oklahoma, USA; ${ }^{3}$ Naomi Berrie Diabetes Center, Columbia University Medical Center, New York, New York, USA; ${ }^{4}$ Department of Pediatrics, Baylor College of Medicine, Houston, Texas, USA; ${ }^{5}$ Department of Pediatrics, Massachusetts General Hospital, Harvard Medical School, Boston, Massachusetts, USA; ${ }^{6}$ Division of Diabetes, Endocrinology and Metabolic Diseases, National Institute of Diabetes and Digestive and Kidney Diseases, National Institutes of Health, Bethesda, Maryland, USA; ${ }^{7}$ Regeneron Genetics Center, Regeneron, Tarrytown, New York, USA; ${ }^{8}$ Department of Pediatrics, Saint Louis University Health Sciences Center, St. Louis, Missouri, USA; ${ }^{9}$ Department of Pediatrics, Washington University School of Medicine, St. Louis, Missouri, USA. Correspondence: Toni I. Pollin (tpollin@som.umaryland.edu)

${ }^{10} \mathrm{~A}$ list of the members of the TODAY Study Group can be found in the online appendix.

Submitted 17 April 2017; accepted 20 July 2017; advance online publication 12 October 2017. doi:10.1038/gim.2017.150
} 
does not have features of T1D (negative for diabetesassociated antibodies) or T2D (nonobese, lacking other metabolic features) especially when there is a strong family history of diabetes, or when there is stable, mildly elevated fasting blood glucose. ${ }^{10}$ However, studies indicate these guidelines are either not utilized or fail to detect many cases of monogenic diabetes. The SEARCH study for diabetes in youth discovered that more than $90 \%$ of patients with $G C K$, $H N F 1 A$, or HNF4A variants were misdiagnosed as T1D (36\%) or T2D (51\%), and only $19 \%$ of patients with MODY variants had treatment appropriate to their etiology. ${ }^{11}$ Many factors contribute to the underdiagnosis of monogenic diabetes, including heterogeneity of monogenic diabetes patient characteristics, similarity between monogenic diabetes and the more common forms of diabetes (especially with increasing prevalence of $\mathrm{T} 2 \mathrm{D}$ in children and adolescents), cost of genetic testing, lack of insurance reimbursement, and lack of awareness among health-care providers. With such high rates of overweight and obesity in young people, one might expect common co-occurrence of a T2D phenotype with monogenic diabetes, making currently suggested algorithms for diagnosis of monogenic diabetes even less sensitive.

Although T2D has been historically found mainly in overweight adults over age 40, recent increases in overweight or obese adolescents have led to an increased occurrence of $\mathrm{T} 2 \mathrm{D}$ in young populations. Because of the increasing prevalence of $\mathrm{T} 2 \mathrm{D}$ in adolescents and the lack of data regarding adolescent-specific T2D treatment methods, the Treatment Options for type 2 Diabetes in Adolescents and Youth (TODAY) study analyzed the effectiveness of metformin alone or in combination with rosiglitazone or lifestyle changes in adolescents with recently diagnosed T2D. ${ }^{12}$ Approximately half $(48.3 \%)$ of the 699 participants treated with metformin alone maintained glycemic control, while the combination of metformin and rosiglitazone showed a small but significant improvement in the durability of glycemic control (61.4\% maintained control, $25.3 \%$ decrease in primary outcome occurrence compared with metformin alone, $P=0.006)$ over a relatively short duration of treatment (patients were followed for an average of 3.86 years). ${ }^{13}$ In the current study, we analyzed the prevalence of monogenic diabetes in the TODAY study participants and their outcomes.

\section{Patient characteristics}

\section{MATERIALS AND METHODS}

The TODAY study participants were adolescents age 10-17 diagnosed with T2D according to American Diabetes Association criteria within 2 years of study enrollment. The protocol for the TODAY study was approved by the institutional review board at each participating institution (ClinicalTrials.gov: NCT00081328), and informed consent was obtained from all subjects. Eligibility criteria also included body mass index (BMI) above the 85th percentile while accounting for age and sex, negative for diabetes-associated autoantibodies (GAD65 and ICA512), and fasting C-peptide $\geq 0.20 \mathrm{nmol} / \mathrm{L}$. Following a run-in period in which glycemic control $(\mathrm{HbAlc}<8 \%)$ on metformin alone was attained, metabolic, glycemic, anthropometric, and lifestyle attributes were collected longitudinally from all TODAY study participants. Patients were followed longitudinally until they lost glycemic control and reached the primary outcome of the study, defined as a glycated hemoglobin value of at least 8.0\% for 6 consecutive months or the inability to wean the participant from insulin within 3 months after treatment for acute metabolic decompensation. Further descriptions of study protocol, design, methods, and results have been previously reported. ${ }^{12,13}$

This study analyzed DNA from a subset of 488 adolescents (177 males and 311 females) from the total TODAY cohort of 699 participants. Some TODAY participants had no DNA available to analyze because they did not attend a study visit during the DNA collection period or because they refused to consent to provide genetic data. In addition, all participants of undefined race/ethnicity or of a race/ethnicity with a small sample size were excluded from this analysis. Subject data included in this analysis were Hispanic $(n=217)$, nonHispanic black (NHB, $n=166$ ), or non-Hispanic white (NHW, $n=105$ ) and showed no obvious differences from the entire TODAY cohort with those race/ethnicities (Supplementary Tables S1 and S2 online).

\section{Sequencing methods}

Coding and flanking regions of 40 autosomal genes with variants known or predicted to cause monogenic diabetes, including 13 genes identified to cause MODY at the time of study design (APPL1 was published as a cause of MODY after the design of our study), as well as genes causing neonatal diabetes, diabetes syndromes, lipodystrophy, severe obesity, and hyperinsulinemia (based on the theory that gain-offunction mutations could cause diabetes), were analyzed by next-generation sequencing using a customized gene panel (Supplementary Table S3). DNA amplification, barcoding, and purification were performed using the Ion AmpliSeq Library Kit 2.0, Ion Xpress Barcode Adapter Kit, and Ion Library Equalizer Kit (Life Technologies, Carlsbad, CA). Emulsion polymerase chain reaction was performed on the Ion One Touch 2 using the Ion PGM Template OT2 200 Kit, and isolation of ion sphere particles with clonally amplified DNA was performed with the Ion Torrent OneTouch ES (Life Technologies). Sequencing was performed using the Ion Torrent Personalized Genome Machine with the Ion PGM Sequencing 200 Kit version 2 and Ion 316 Chip version 2 (Life Technologies). Alignment was performed using TMAP version 4.2.14 software (https://github.com/iontorrent/ TMAP/releases). Variant calling was performed using the Torrent Suite variantCaller plugin version $4.2-14$ and coverage analysis was performed using the Torrent Suite coverageAnalysis plugin version 4.2. Only samples with $\geq 20 \times$ mean base coverage depth of $\geq 80 \%$ of the target region $(139,491 \mathrm{bp})$ were used for analysis. Single-nucleotide variants with genotype quality scores $<20$ and coverage 
depth $<10 \times$ were filtered from analysis, as were insertions/ deletions with genotype quality scores $<50$ and coverage depth $<50 \times$. Samples had a mean of 186,170 $\pm 67,019$ (SD) mapped reads, and the single-nucleotide variant transition to transversion ratio was 2.31. Quality metrics of monogenic diabetes variants are included in Supplementary Table S4 online. Variants were annotated using a customized pipeline using multiple large population data sets, in silico prediction tools, and conservation metrics. All variants in noncoding or noncanonical splice regions, variants with a minor allele frequency $>5.0 \%$, or synonymous variants were filtered from further analysis.

\section{Variant analysis}

Noncommon ( $<5 \%$ minor allele frequency) coding or splicesite variants were analyzed for pathogenicity according to American College of Medical Genetics and Genomics/ Association for Molecular Pathology (ACMG/AMP) guidelines for variant interpretation. ${ }^{14}$ These guidelines were created to standardize the complex process of classifying variants into categories ("pathogenic," "likely pathogenic," "uncertain significance," "likely benign," or "benign") based on population data, computational data, functional data, and segregation data. Criteria (Supplementary Table S5) were assessed for each variant and pathogenicity was determined based on the total evidence according to the algorithm of the ACMG/AMP guidelines. ${ }^{14}$

\section{Statistical analysis}

Patients were grouped for statistical analysis. The unaffected group $(n=426)$ did not have any pathogenic, likely pathogenic, or previously cited monogenic diabetes variants. Other groups included patients with pathogenic or likely pathogenic variants in any monogenic diabetes gene $(n=22)$, HNF1A $(n=5)$, HNF4A $(n=7)$, or GCK $(n=7)$. Participants with previously cited monogenic diabetes variants that were not classified as pathogenic or likely pathogenic $(n=40)$ were not included in analysis due to uncertainty over their monogenic diabetes status. Associations between monogenic diabetes subcategories and patient characteristics, including BMI $z$-score, $\mathrm{HbAlc}$, fasting glucose, fasting insulin, insulinogenic index, dual energy X-ray absorptiometry (DXA), lipid measures, and blood pressure, were evaluated using linear models accounting for sex, age, race, and BMI $z$ score (where indicated). These measures were collected from the earliest available time-point for the trait (screening data for those traits where it was available; otherwise from baseline measures). Log transformation was used to normalize data sets with skewed distributions. Because these analyses were hypothesis-driven, a $P$ value of $<0.05$ was considered nominally significant and no adjustment for multiple comparison testing was performed. ${ }^{15}$ Treatment failure was defined as the primary outcome of the TODAY study (loss of glycemic control, defined as a glycated hemoglobin values of at least $8.0 \%$ for 6 consecutive months or the inability to wean the participant from insulin within 3 months after treatment for acute metabolic decompensation). Treatment failure analyses were performed using a Cox proportional hazards model using the patient outcomes specified in the original TODAY study. ${ }^{13}$ The treatment failure analysis accounted for participant sex, age, race/ethnicity, and TODAY treatment group. The proportional hazards assumption was met by each variable, except for sex, which was treated as a stratifying variable in the model.

\section{RESULTS}

\section{Monogenic diabetes gene variants}

The 13 genes reported to cause MODY at the time of study design were analyzed for noncommon coding and splice-site variants in this cohort (Supplementary Table S6). Twenty-six previously cited MODY variants were assessed according to ACMG/AMP criteria, and 18 were classified pathogenic or likely pathogenic (Table 1 ), while eight were classified as benign, likely benign, or variants of uncertain significance (VUS) (Supplementary Table S7). Thirty novel variants were discovered, and four of those variants were classified as pathogenic or likely pathogenic, while 26 were VUS (Supplementary Table S8). Patients with pathogenic or likely pathogenic variants were considered to have monogenic diabetes. In sum, 22 individuals with 22 different pathogenic or likely pathogenic variants were discovered; i.e., $22 / 488$ of those analyzed, or $4.5 \%$ of this sample of TODAY participants, had monogenic diabetes. Patients with monogenic diabetes were found across each of the three categories of race/ethnicity and across all treatment arms of the TODAY clinical trial (Table 1, Supplementary Table S2). While variants were found in non-MODY genes in this study, none were determined to be pathogenic or likely pathogenic for monogenic diabetes or monogenic forms of obesity (Supplementary Table S9).

\section{Characteristics of patients with monogenic diabetes gene variants and study outcomes}

Characteristics at the earliest available time-point (BMI $z$ score, HbAlc, blood pressure, triglycerides, and lipid measures were from the screening visit, while fasting glucose, fasting insulin, insulinogenic index, and DXA measures were from the baseline visit) of subjects with pathogenic or likely pathogenic monogenic diabetes variants $(n=22)$ were compared with those without monogenic diabetes $(n=426)$ (Table 2, Supplementary Table S10). Individuals with monogenic diabetes showed lower BMI $z$-scores (2.05 vs. 2.32, $P=0.004)$, higher fasting glucose (6.65 vs. $6.08 \mathrm{mmol} / \mathrm{L}$, $P=0.02)$, lower fasting insulin (152.1 vs. $213.9 \mathrm{pmol} / \mathrm{L}$, $P=0.03)$, and higher total cholesterol (4.50 vs. $3.98 \mathrm{mmol} / \mathrm{L}$, $P=0.003)$ compared with individuals without monogenic diabetes. These associations were still significant when adjusted for BMI $z$-score (Supplementary Table S10). Separated by gene etiology, monogenic diabetes subgroups showed similar trends in patient characteristics, although they were generally underpowered to detect significant differences. 


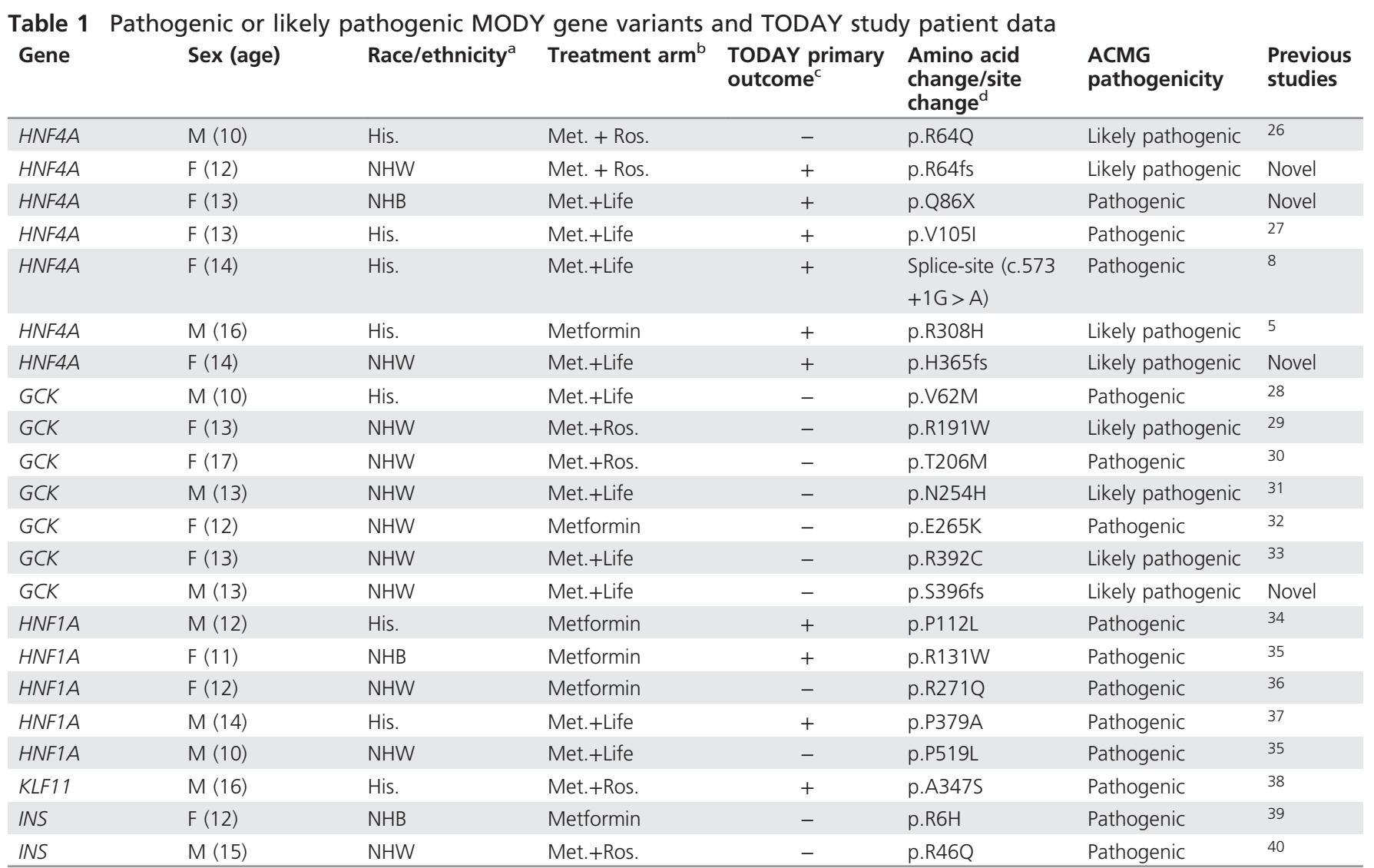

ACMG, American College of Medical Genetics and Genomics; F, female; His., Hispanic; M, male; MODY, maturity-onset diabetes of the young; NHB, non-Hispanic black; NHW, non-Hispanic white; TODAY, Treatment Options for type 2 Diabetes in Adolescents and Youth.

Quality metrics for each variant can be found in Supplementary Table S4.

aSelf-reported race/ethnicity. ${ }^{\mathrm{b}}$ TODAY clinical trial treatment arm: Met.+Ros., metformin and rosiglitazone; Met.+Life, metformin and lifestyle intervention. ${ }^{\mathrm{C}}$ TODAY clinical trial outcome: $(-)$, patient did not reach primary outcome (treatment failure); $(+)$, patient reached primary outcome (treatment failure). ${ }^{d}$ Amino acid reported sites are according to the following reference sequences: HNF4A-NM_001287183; NP_001274112, GCK-NM_000162; NP_000153, HNF1A-NM_000545; NP_000536, KLF11NM_003597; NP_003588, and INS-NM_000207; NP_000198.1.

Time-to-treatment failure analyses, the primary outcome of the TODAY study, compared unaffected individuals $(n=426)$ with individuals with HNF1A-MODY, HNF4AMODY, and GCK-MODY (Figure 1). No patients with GCKMODY (0/7) failed treatment in the TODAY study. Across all three study arms, 6 of 7 of the subjects with HNF4A-MODY failed treatment over the first 2 years of study, with a hazard ratio of $5.03 \quad(2.18-11.58,95 \%$ confidence interval) $(P=0.0002)$ compared with subjects without monogenic diabetes. Three patients with HNF4A-MODY lost glycemic control by their first postbaseline study visit. There was no significant difference in treatment response of individuals with HNF1A-MODY compared with individuals without monogenic diabetes.

\section{DISCUSSION}

Based on our findings, an appreciable number of youth diagnosed with T2D may, in fact, have undiagnosed monogenic diabetes. Individuals with monogenic diabetes participating in TODAY displayed subtle, but significant, differences in select characteristics compared with unaffected TODAY participants. However, in this adolescent population sample selected for being overweight or obese and having nonautoimmune, C-peptide-positive diabetes, it was not possible to reliably distinguish between T2D and monogenic diabetes based on clinical features at baseline in this study. Importantly, patients with monogenic diabetes were found in each race/ethnicity examined, emphasizing that race/ethnicity should not be used to discriminate between those with and without monogenic diabetes. We also confirmed the importance of a genetic diagnosis of monogenic diabetes to inform treatment protocols since metformin, while the first-line treatment of T2D, was ineffective in those who turned out to have HNF4-MODY. Further emphasizing the known relationship between genetic diagnosis and established treatment protocols, those with GCK-MODY did not fail treatment, since patients with GCK-MODY do not generally have highly elevated HbA1c and typically do not require drug therapy. Both of these findings have strong implications for clinical practice.

At least $4.5 \%$ of TODAY participants (22/488) had pathogenic or likely pathogenic monogenic diabetes variants (Table 1). It is estimated that there are approximately 3,700 new diagnoses of T2D in youth each year in the United States, 
Table 2 Associations between all monogenic diabetes variants, gene subtypes, and patient characteristics at earliest study time-point (screening or baseline), adjusted for age, sex, and race/ethnicity

Unaffected All monogenic HNF4A -MODY GCK-MODY HNF1A-MODY INS-MODY diabetes $^{\mathrm{a}}$

\begin{tabular}{|c|c|c|c|c|c|c|c|}
\hline & & & & & & & \\
\hline$n$ & 426 & 22 & 7 & 7 & 5 & 2 & 1 \\
\hline BMI $z$-score Mean $\pm S D$ & $2.32 \pm 0.42$ & $2.05 \pm 0.42^{b}$ & $2.12 \pm 0.52$ & $1.91 \pm 0.33^{b}$ & $2.06 \pm 0.32$ & $2.52 \pm 0.44$ & 1.49 \\
\hline DXA fat $(\%)^{c}$ & $38.3 \pm 6.02$ & $35.2 \pm 6.9$ & $38.4 \pm 7.0$ & $38.3 \pm 5.7$ & $29.9 \pm 1.5^{b}$ & 30.6 & 23.9 \\
\hline $\mathrm{HbA1c}(\%)$ & $7.51 \pm 1.97$ & $7.45 \pm 1.93$ & $7.27 \pm 2.35$ & $6.99 \pm 2.23$ & $7.78 \pm 1.03$ & $9.40 \pm 0.57$ & 6.4 \\
\hline Fasting glucose (mmol/L) & $6.08 \pm 1.27$ & $6.65 \pm 1.56^{b}$ & $7.15 \pm 1.09^{b}$ & $6.69 \pm 0.28$ & $5.46 \pm 1.2$ & $5.22 \pm 0.24$ & 11.6 \\
\hline Fasting insulin (pmol/L) & $213.9 \pm 145.3$ & $152.1 \pm 210.0^{b}$ & $122.2 \pm 46.3$ & $87.5 \pm 32.7^{b}$ & $107.6 \pm 7.5^{b}$ & $588.9 \pm 679.7$ & 164.6 \\
\hline $\begin{array}{l}\text { Insulinogenic index } \\
\left(\operatorname{Ins}_{30-0} / \mathrm{Glu}_{30-0}\right)\end{array}$ & $1.63 \pm 2.45$ & $0.81 \pm 0.84$ & $0.56 \pm 0.41$ & $1.28 \pm 1.24$ & $0.49 \pm 0.17$ & 1.29 & 0.08 \\
\hline $\begin{array}{l}\text { Diastolic blood pressure } \\
(\mathrm{mmHg})\end{array}$ & $67.2 \pm 8.8$ & $64.3 \pm 9.7$ & $67.1 \pm 12.0$ & $60.9 \pm 11.3$ & $64.7 \pm 5.4$ & $66.3 \pm 8.1$ & 63.5 \\
\hline Total cholesterol (mmol/L) & $3.98 \pm 0.86$ & $4.50 \pm 1.06^{b}$ & $4.77 \pm 1.15^{b}$ & $4.11 \pm 0.9$ & $4.38 \pm 1.08$ & $4.26 \pm 0.68$ & 6.4 \\
\hline HDL cholesterol (mmol/L) & $1.03 \pm 0.24$ & $1.09 \pm 0.24$ & $1.19 \pm 0.21$ & $1.05 \pm 0.34$ & $1.03 \pm 0.12$ & $1.18 \pm 0.20$ & 0.83 \\
\hline
\end{tabular}

BMI, body mass index; DXA, dual energy X-ray absorptiometry; HDL, high-density lipoprotein; LDL, low-density lipoprotein; MODY, maturity-onset diabetes of the young; TODAY, Treatment Options for type 2 Diabetes in Adolescents and Youth.

Values are presented as mean \pm SD. All measures were taken at screening in the TODAY study, except for fasting glucose, fasting insulin, insulinogenic index, and DXA fat measures, which were taken at the baseline time-point.

${ }^{a}$ Monogenic diabetes pathogenic or likely pathogenic mutation. ${ }^{b} P<0.05$ for the effect size of the classification (monogenic diabetes status in aggregate or separated by gene) in a linear model accounting for sex, age, and race/ethnicity (effect sizes, BMI $Z$-score adjusted results, and $P$ values found in Supplementary Table S9). 'DXA data was available for 303 unaffected individuals and 15 patients with monogenic diabetes (5 HNF4A-MODY, 5 GCK-MODY, 3 HNF1A-MODY, 1 INS-MODY, and 1 KLF11-MODY).

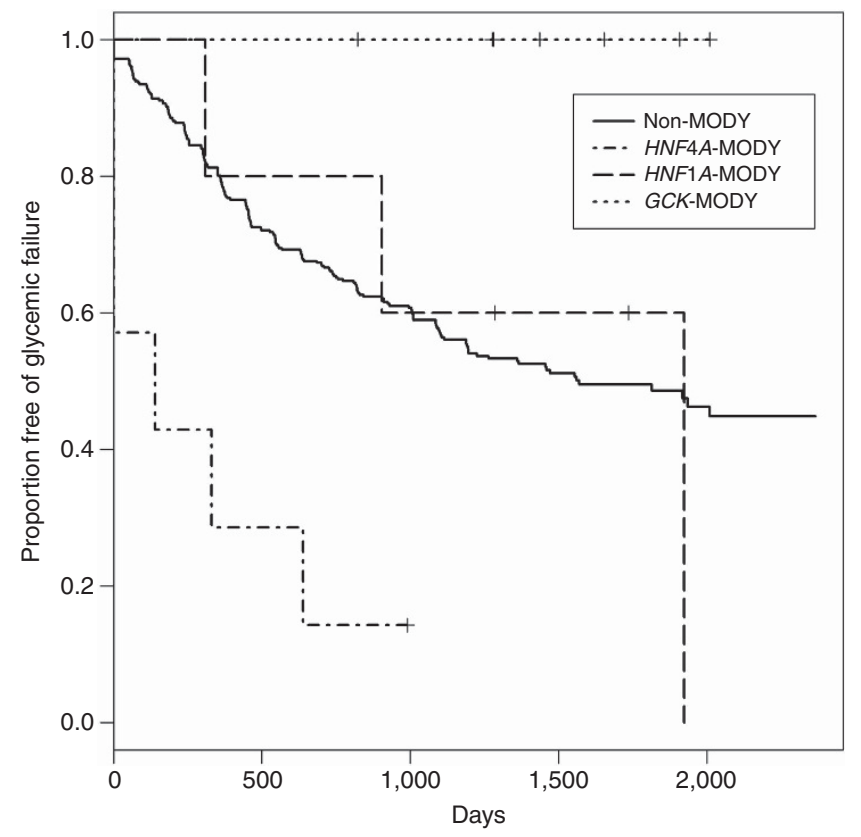

Figure 1 Failure-free survival curve of maturity-onset diabetes of the young (MODY) gene subtypes and unaffected patients. $X$-axis is defined as post-baseline visit days until start of failure interval. Events are defined as elevated glycated hemoglobin $(>8.0 \%)$ over a period of 6 months or the inability to wean the participant from insulin within 3 months after treatment initiation in the Treatment Options for type 2 Diabetes in Adolescents and Youth (TODAY) study. Hazard ratio (HR) for each subtype: GCK-MODY HR = undefined (no events), HNF1A-MODY $\mathrm{HR}=1.26(0.40-4.0295 \%$ confidence interval) $P=0.7$, and HNF4AMODY HR $=5.03(2.18-11.5895 \%$ confidence interval) $P=0.0002$. and our findings suggest as many as 160 of those cases could be misdiagnosed cases of monogenic diabetes. ${ }^{16}$ The SEARCH study for diabetes in youth previously found $8 \%$ of participants with diabetes-associated autoantibody-negative, C-peptide-positive diabetes had monogenic diabetes variants in GCK, HNF1A, and HNF4A. ${ }^{11}$ Similar to the SEARCH study, we found no differences in family history of diabetes, as measured by reported maternal and paternal diabetes status, between TODAY patients with or without monogenic diabetes, and we found comparable racial/ethnic distributions of patients with monogenic diabetes between TODAY and SEARCH (Supplementary Table S2). We probably found a lower percentage of patients with monogenic diabetes because the TODAY study eligibility criteria required adolescents with diabetes to be overweight or obese. Additionally, it is possible for individuals to have monogenic diabetes and coincident insulin resistance or T2D, which can further complicate both diagnosis and treatment. While likely to only account for a minority of cases, patients with authentic T1D or T2D harboring monogenic diabetes variants have been described before. $^{17,18}$

By following the ACMG/AMP guidelines for variant annotation, our study has potentially limited our estimation of the prevalence of monogenic diabetes in the cohort. While the ACMG/AMP guidelines are important for correctly classifying clinical implications of variants before being returned to patients, the need for evidence across multiple categories (e.g., functional studies, family cosegregation data, de novo status) can be restrictive, especially for recently 
discovered variants. As shown in previous studies, this standardized variant classification process often reclassifies variants previously assumed to be pathogenic as benign, likely benign, or VUS due to increases in genetic and phenotypic database information. ${ }^{19}$ Patients with "reclassified" variants were excluded from our statistical analysis, but separate analysis of clinical characteristics of carriers of the $B L K$ p.A71T and KLF11 p.T220M variants showed no differences from those without monogenic diabetes (data not shown). Analysis of 18 VUS discovered (11 novel variants) in the three most common MODY genes showed patients with VUS were intermediate or close to the monogenic diabetes group in terms of clinical characteristics (BMI $z$-score $=2.27 \pm 0.48$, fasting glucose $=6.72 \pm 1.57 \mathrm{mmol} / \mathrm{L}$, fasting insulin $=173 \pm$ $123 \mathrm{pmol} / \mathrm{L}$, mean $\pm \mathrm{SD}$ ), but differences from those without monogenic diabetes ( $n=412$ in this analysis) were not statistically significant. This finding supports the hypothesis that at least some of the VUS may cause monogenic diabetes. Since the ACMG/AMP guidelines often classify novel variants as VUS due to the lack of evidence to suggest the variant is either pathogenic or benign, further study of the novel variants found in this study is a potentially fruitful topic for future research and could increase the estimate of monogenic diabetes prevalence in this study.

Although pathogenic and likely pathogenic monogenic diabetes genetic variants were found in each race/ethnicity, a higher proportion of NHW participants carried monogenic diabetes variants (Supplementary Table S2). Similarly, more NHB participants carried previously cited monogenic diabetes variants reclassified as VUS, likely benign, or benign variants. The odds ratio for NHB participants carrying reclassified variants was 2.15 compared with NHW, 3.84 compared with Hispanic, and 3.10 compared with the combined NHW and Hispanic populations. We hypothesize this trend is due to the reliance of the ACMG/AMP standards and guidelines on previously published data on genetic variants. Previous monogenic diabetes studies have mainly focused on European populations, which could cause bias in classifying variants. Underrepresentation of minority populations in genetic studies is a well-known concern that must be addressed for precision genetic medicine to be effective across all races/ethnicities. At this point, it is important to recognize that monogenic diabetes variants can and have been found across multiple different races/ethnicities.

Through the design of the gene panel, we have potentially limited discovery of patients with monogenic diabetes in this cohort. Since there have been relatively few reported pathogenic variants in the 27 non-MODY genes compared with the number of reported pathogenic MODY gene variants, coding variants in non-MODY genes are less likely to be classified as pathogenic or likely pathogenic. Although the discovery of more pathogenic or likely pathogenic variants in less common monogenic diabetes genes may increase in the future, because we were unable to define any likely pathogenic or pathogenic variants in non-MODY monogenic diabetes genes, MODY-specific gene panels may be a more appropriate approach for studies screening for monogenic diabetes at this time. Also, there are potentially other unknown causative monogenic diabetes genes or nonexonic/splicing variants not assessed by using our gene panel.

We found a larger proportion of individuals with HNF4AMODY than expected in our cohort (Table 1). While HNF1AMODY accounts for approximately 30-50\% of MODY diagnoses and HNF4A-MODY accounts for less than $10 \%$, we observed more patients with HNF4A-MODY than with HNF1A-MODY. ${ }^{20}$ Interestingly, HNF4A-MODY has been associated with increased birth weight and macrosomia in the neonatal stage, regardless of maternal genotype but exacerbated by the mother having the same mutation and the associated hyperglycemic intrauterine environment. ${ }^{21}$ Macrosomia has been correlated with overweight or obese status through adolescence and adulthood. ${ }^{22}$ Thus there could be an association between HNF4A-MODY with higher BMI that could cause HNF4A-MODY to be misdiagnosed as T2D when using $\mathrm{BMI}$ as a criterion for monogenic diabetes. We hypothesize that the TODAY study inclusion criteria (BMI $\geq 85$ th percentile for age and sex) may have created a selection bias toward HNF4A-MODY compared with the other genespecific subgroups; however, this remains to be demonstrated. Further studies incorporating birth weight and prevalence of T2D misdiagnosis of patients with HNF4A-MODY are necessary to test this hypothesis.

We did not discover any clinical criteria to differentiate overweight or obese adolescents with monogenic diabetes from those with T2D in this cohort selected for overweight or obese status and nonautoimmune, C-peptide-positive diabetes. Individuals with monogenic diabetes had lower BMI $z$ score $(P=0.004)$, but all 22 adolescents with monogenic diabetes were still overweight ( $>85$ th percentile by age) per the TODAY study design. For each of the clinical characteristics measured in the TODAY study, values of patients with monogenic diabetes could be found throughout the range of values for patients with T2D (Table 2). The functional effects of genetic defects leading to an insulin secretion deficit were demonstrated as adolescents with monogenic diabetes had lower fasting plasma insulin $(P=0.03)$ compared with those without monogenic diabetes. They also had higher fasting blood glucose $(P=0.02)$ concentrations. Similar to published studies on populations with broader patient demographics, our patients with monogenic diabetes have greater insulin sensitivity (defined as the inverse of the fasting insulin) compared with those in our cohort with apparent T2D, but characteristics such as high-density lipoprotein cholesterol and beta cell function (insulinogenic index) were not different between the groups in this specific cohort selected by overweight/obese status and nonautoimmune diabetes. ${ }^{23-25}$ In summary, within this overweight/obese autoantibodynegative, C-peptide-positive diabetic cohort, there were differences in the means of some metabolic characteristics, but it was not possible to identify any characteristics to reliably distinguish between those with and without monogenic etiologies. Other markers shown to help identify 
patients with monogenic diabetes, such as high-sensitivity C-reactive protein and plasma glycan profile, were not collected in the TODAY study.

Although the TODAY study provided unique information about the effectiveness of metformin alone or in combination with rosiglitazone or lifestyle changes, the small numbers of patients with each gene subtype of monogenic diabetes reduced our ability to draw conclusions regarding response to each of the treatment arms. However, none of the patients with GCK-MODY failed the treatment regimens in the TODAY study. This is consistent with the GCK-MODY phenotype of mildly elevated fasting blood glucose (fasting glucose of $5.49-8.66 \mathrm{mmol} / \mathrm{L}$ and $\mathrm{HbAlc}$ of $5.6-7.6 \%)$ that usually needs no treatment to avoid chronic complications of diabetes. In contrast, 6 of 7 patients with HNF4A-MODY failed treatment across study arms (hazard ratio $=5.03 P=0.0002$ ), indicating poor response regardless of therapies offered in the TODAY study. Similarly, though not statistically significant, 3/5 patients with HNF1A-MODY failed the TODAY study treatments. These results would be expected since the established treatment for HNF1A- and HNF4A-MODY are sulfonylurea drugs, rather than metformin and/or rosiglitazone. ${ }^{7,8}$ Metformin is an insulin-sensitizing agent, while sulfonylureas are insulin secretagogues that improve the insulin secretion deficit found in HNF1A- and HNF4AMODY patients. Therefore the finding that HNF4A-MODY patients failed metformin treatments in the TODAY trial is a demonstration of the consequences of not attaining a genetic diagnosis of MODY. We note that it is possible that in addition to sulfonylureas, metformin and/or thiazolidinediones may be appropriate for some monogenic diabetes patients with concomitant obesity and insulin resistance.

Genetic testing is not commonly implemented in diabetes clinical care due to current costs of testing, uncertainty over insurance reimbursement, and difficulty of interpretation of sequencing results. However, clinical care is moving into an era of genomic medicine, and monogenic diabetes provides a unique opportunity for immediate implementation of personalized genomic medicine. Under specific conditions of cost and discovery rate, genetic testing for MODY has been modeled to be as cost-effective as current medical practices and potentially cost-saving. ${ }^{6}$ As the cost and throughput of genetic testing continue to improve, the knowledge base of rare genetic variation will continue to grow to inform clinical practice. Although current impediments to genetic testing such as cost, availability, and reimbursement may limit genetic testing for monogenic diabetes in large populations, this study has shown monogenic diabetes should be considered as a possible diagnosis in young people with antibody-negative and C-peptide-positive diabetes. However, it is also important to recognize that $\mathrm{T} 2 \mathrm{D}$ is the most common form of diabetes in the world, and most overweight/ obese individuals with diabetes will have T2D, especially those not diagnosed at a young age ( $>30$ years old).

The findings from this study have strong implications for informing the practice of managing diabetes in youth. We discovered individuals with monogenic diabetes across all races/ethnicities in a cohort of overweight and obese adolescents diagnosed with $\mathrm{T} 2 \mathrm{D}$, raising concerns about the currently recommended use of BMI and previously recommended use of race/ethnicity to select patients for genetic testing. We suggest that with secular trends of increasing obesity in children and adolescents, monogenic diabetes be considered as a potential etiology in diabetes-associated autoantibody-negative and C-peptide-positive adolescents regardless of BMI. Despite the small sample size of our cohort, treatment response based on monogenic diabetes diagnosis was consistent with predicted results, indicating the importance of monogenic diabetes genetic testing and proper genetic interpretation for providing optimal treatment to youth with diabetes.

\section{SUPPLEMENTARY MATERIAL}

Supplementary material is linked to the online version of the paper at http://www.nature.com/gim

\section{ACKNOWLEDGMENTS}

The TODAY Study Group thanks the following companies for donations in support of the efforts of the clinical trial: Becton, Dickinson and Company; Bristol-Myers Squibb; Eli Lilly and Company; GlaxoSmithKline; LifeScan; Pfizer; and Sanofi. This work was completed with funding from the National Institute of Diabetes and Digestive and Kidney Diseases and the National Institutes of Health Office of the Director through grants U01DK-61212, U01-DK-61230, U01-DK-61239, U01-DK-61242, and U01-DK-61254; from the National Center for Research Resources General Clinical Research Centers Program grants M01-RR-00036 (Washington University School of Medicine in St. Louis), M01RR00043-45 (Children's Hospital Los Angeles), M01-RR00069 (University of Colorado Denver), M01-RR00084 (Children's Hospital of Pittsburgh), M01-RR01066 (Massachusetts General Hospital), M01-RR00125 (Yale University), and M01-RR14467 (University of Oklahoma Health Sciences Center); from the National Center for Research Resources Clinical and Translational Science Awards UL1-RR-024134 (Children's Hospital of Philadelphia), UL1-RR-024139 (Yale University), UL1-RR-024153 (Children's Hospital of Pittsburgh), UL1-RR-024989 (Case Western Reserve University), UL1-RR-024992 (Washington University in St. Louis), UL1-RR-025758 (Massachusetts General Hospital), and UL1-RR-025780 (University of Colorado Denver); from the National Center for Advancing Translational Sciences grant UL1TR-000448 (Washington University); and from National Human Genome Research Institute grant U01-HG-007775 (University of Maryland).

\section{DISCLOSURE}

The authors declare no conflict of interest.

\section{REFERENCES}

1. Tattersall RB, Fajans SS. A difference between the inheritance of classical juvenile-onset and maturity-onset type diabetes of young people. Diabetes. 1975;24:44-53. 
2. Murphy R, Ellard S, Hattersley AT. Clinical implications of a molecular genetic classification of monogenic beta-cell diabetes. Nat Clin Pract Endocrinol Metab. 2008;4:200-213.

3. Shepherd M, Shields B, Hammersley S, et al. Systematic population screening, using biomarkers and genetic testing, identifies $2.5 \%$ of the U.K. pediatric diabetes population with monogenic diabetes. Diabetes Care. 2016;39:1879-1888.

4. Weinreich SS, Bosma A, Henneman L, et al. A decade of molecular genetic testing for MODY: a retrospective study of utilization in the Netherlands. Eur J Hum Genet. 2015;23:29-33.

5. Delvecchio M, Ludovico O, Menzaghi $C$, et al. Low prevalence of HNF1A mutations after molecular screening of multiple MODY genes in 58 Italian families recruited in the pediatric or adult diabetes clinic from a single Italian hospital. Diabetes Care. 2014;37:e258-60.

6. Naylor RN, John PM, Winn AN, et al. Cost-effectiveness of MODY genetic testing: translating genomic advances into practical health applications. Diabetes Care. 2014;37:202-209.

7. Shepherd M, Shields B, Ellard S, Rubio-Cabezas O, Hattersley AT. A genetic diagnosis of HNF1A diabetes alters treatment and improves glycaemic control in the majority of insulin-treated patients. Diabet Med. 2009;26:437-441.

8. Pearson ER, Pruhova S, Tack CJ, et al. Molecular genetics and phenotypic characteristics of MODY caused by hepatocyte nuclear factor 4alpha mutations in a large European collection. Diabetologia. 2005;48: 878-885.

9. Steele AM, Shields BM, Wensley KJ, Colclough K, Ellard S, Hattersley AT. Prevalence of vascular complications among patients with glucokinase mutations and prolonged, mild hyperglycemia. JAMA. 2014;311: 279-286.

10. American Diabetes Association. 2. Classification and diagnosis of diabetes. Diabetes Care. 2017;40(suppl 1):S11-S24.

11. Pihoker C, Gilliam LK, Ellard S, et al. Prevalence, characteristics and clinical diagnosis of maturity onset diabetes of the young due to mutations in HNF1A, HNF4A, and glucokinase: results from the SEARCH for diabetes in youth. J Clin Endocrinol Metab. 2013;98:4055-4062.

12. TODAY Study Group, Zeitler P, Epstein L, et al. Treatment options for type 2 diabetes in adolescents and youth: a study of the comparative efficacy of metformin alone or in combination with rosiglitazone or lifestyle intervention in adolescents with type 2 diabetes. Pediatr Diabetes. 2007;8:74-87.

13. TODAY Study Group, Zeitler $P$, Hirst $K$, et al. A clinical trial to maintain glycemic control in youth with type 2 diabetes. N Engl J Med. 2012;366: 2247-2256.

14. Richards S, Aziz N, Bale S, et al. Standards and guidelines for the interpretation of sequence variants: a joint consensus recommendation of the American College of Medical Genetics and Genomics and the Association for Molecular Pathology. Genet Med. 2015;17:405-423.

15. Rothman KJ. No adjustments are needed for multiple comparisons. Epidemiology. 1990;1:43-46.

16. Writing Group for the SEARCH for Diabetes in Youth Study Group, Dabelea D, Bell RA, et al. Incidence of diabetes in youth in the United States. JAMA. 2007;297:2716-2724.

17. McDonald TJ, Colclough K, Brown R, et al. Islet autoantibodies can discriminate maturity-onset diabetes of the young (MODY) from type 1 diabetes. Diabet Med. 2011;28:1028-1033.

18. Wheeler BJ, Patterson N, Love DR, et al. Frequency and genetic spectrum of maturity-onset diabetes of the young (MODY) in southern New Zealand. J Diabetes Metab Disord. 2013;12:46-6581-12-46.

19. Chambers C, Fouts A, Dong F, et al. Characteristics of maturity onset diabetes of the young in a large diabetes center. Pediatr Diabetes. 2015:17:360-7.

20. Shields BM, Hicks S, Shepherd MH, Colclough K, Hattersley AT, Ellard S. Maturity-onset diabetes of the young (MODY): how many cases are we missing? Diabetologia. 2010;53:2504-2508.
21. Pearson ER, Boj SF, Steele AM, et al. Macrosomia and hyperinsulinaemic hypoglycaemia in patients with heterozygous mutations in the HNF4A gene. PLoS Med. 2007;4:e118.

22. Wang Y, Gao E, Wu J, et al. Fetal macrosomia and adolescence obesity: results from a longitudinal cohort study. Int J Obes (Lond). 2009;33: 923-928

23. Stride $A$, Ellard $S$, Clark $P$, et al. Beta-cell dysfunction, insulin sensitivity, and glycosuria precede diabetes in hepatocyte nuclear factor-1alpha mutation carriers. Diabetes Care. 2005;28:1751-1756.

24. Fendler $\mathrm{W}$, Rizzo $\mathrm{M}$, Borowiec $\mathrm{M}$, et al. Less but better: cardioprotective lipid profile of patients with GCK-MODY despite lower HDL cholesterol level. Acta Diabetol. 2014;51:625-632.

25. McDonald TJ, McEneny J, Pearson ER, et al. Lipoprotein composition in HNF1A-MODY: differentiating between HNF1A-MODY and type 2 diabetes. Clin Chim Acta. 2012:413:927-932.

26. Forlani G, Zucchini S, Di Rocco A, et al. Double heterozygous mutations involving both HNF1A/MODY3 and HNF4A/MODY1 genes: a case report. Diabetes Care. 2010;33:2336-2338.

27. Monney CT, Kaltenrieder V, Cousin P, Bonny C, Schorderet DF. Large family with maturity-onset diabetes of the young and a novel V121I mutation in HNF4A. Hum Mutat. 2002:20:230-231.

28. Gloyn AL, Noordam K, Willemsen MA, et al. Insights into the biochemical and genetic basis of glucokinase activation from naturally occurring hypoglycemia mutations. Diabetes. 2003;52:2433-2440.

29. Ellard S, Beards F, Allen LI, et al. A high prevalence of glucokinase mutations in gestational diabetic subjects selected by clinical criteria. Diabetologia. 2000;43:250-253.

30. Bertini C, Maioli M, Fresu P, Tonolo G, Pirastu M, Maioli M. A new missense mutation in the glucokinase gene in an Italian MODY family. Diabetologia. 1996;39:1413-1414.

31. Osbak KK, Colclough K, Saint-Martin C, et al. Update on mutations in glucokinase (GCK), which cause maturity-onset diabetes of the young, permanent neonatal diabetes, and hyperinsulinemic hypoglycemia. Hum Mutat. 2009;30:1512-1526.

32. Galan M, Vincent $O$, Roncero I, et al. Effects of novel maturityonset diabetes of the young (MODY)-associated mutations on glucokinase activity and protein stability. Biochem J. 2006;393(pt 1): 389-396.

33. Hattersley AT, Beards F, Ballantyne E, Appleton M, Harvey R, Ellard S. Mutations in the glucokinase gene of the fetus result in reduced birth weight. Nat Genet. 1998;19:268-270.

34. Bjorkhaug L, Ye H, Horikawa Y, Sovik O, Molven A, Njolstad PR. MODY associated with two novel hepatocyte nuclear factor-1alpha loss-offunction mutations (P112L and Q466X). Biochem Biophys Res Commun. 2000:279:792-798.

35. Frayling TM, Bulamn MP, Ellard S, et al. Mutations in the hepatocyte nuclear factor-1alpha gene are a common cause of maturity-onset diabetes of the young in the U.K. Diabetes. 1997:46:720-725.

36. Balamurugan K, Bjorkhaug L, Mahajan S, et al. Structure-function studies of HNF1A (MODY3) gene mutations in South Indian patients with monogenic diabetes. Clin Genet. 2016;90:486-495.

37. Ekholm E, Nilsson R, Groop L, Pramfalk C. Alterations in bile acid synthesis in carriers of hepatocyte nuclear factor 1alpha mutations. J Intern Med. 2013;274:263-272.

38. Neve B, Fernandez-Zapico ME, Ashkenazi-Katalan $\mathrm{V}$, et al. Role of transcription factor KLF11 and its diabetes-associated gene variants in pancreatic beta cell function. Proc Natl Acad Sci U S A. 2005;102: 4807-4812.

39. Boesgaard TW, Pruhova $S$, Andersson EA, et al. Further evidence that mutations in INS can be a rare cause of maturity-onset diabetes of the young (MODY). BMC Med Genet. 2010;11:42-2350-11-42.

40. Molven A, Ringdal M, Nordbo AM, et al. Mutations in the insulin gene can cause MODY and autoantibody-negative type 1 diabetes. Diabetes. 2008;57:1131-1135. 